\title{
Discrimination des crânes du campagnol des champs, Microtus arvalis (Pallas) et du campagnol souterrain, Pitymys subterraneus (de Sélys Longchamps) en l'absence de mandibule
}

par

\author{
Roland M. LIBOIS
}

Pour aboutir à une discrimination des crânes de campagnol des champs, Microtus arvalis (Pallas) et de campagnol souterrain, Pitymys subterraneus (de Sélys Longchamps) lorsque la mandibule est absente, 1799 crânes de $M$. arvalis et 535 crânes de $P$. subterraneus appartenant à différentes populations belges ont été étudiés sous trois aspects :

- La forme des sutures nasal-frontal et prémaxillaire-frontal. Elle constitue un excellent caractère discriminatoire entre les deux espèces et ne souffre que de peu d'exceptions.

- La valeur des rapports hauteur du crâne/longueur du diastème. Elle ne semble être discriminatoire pour $M$. arvalis qu'au-dessus d'un rapport de $95 \%$ et pour $P$. subterraneus qu'en dessous d'un rapport de $86 \%$.

- La largeur de la constriction interorbitaire. C'est également un bon critère d'identiflcation, meme pour de jeunes individus semble-t-il. Cependant, elle est l'objet en Europe d'une variation d'ordre clinal, quoique cette hypothèse mériterait d'étre vérifiée. Pour les populations belges étudiées, ses valeurs s'échelonnent chez $M$. arvalis de 2,60 à $3,50 \mathrm{~mm}$ (moyenne : 3,09 $\mathrm{mm}$ ), chez $P$. subterraneus de 3,30 à $4,00 \mathrm{~mm}$ (moyenne $: 3,65 \mathrm{~mm}$ )

\section{INTRODUGTION}

La discrimination du campagnol des champs, Microtus arvalis (Pallas) et du campagnol souterrain, Pitymys subterraneus (de Sélys Longchamps) ne pose guère de problèmes lorsqu'on dispose des mandibules. On sait que la première molaire (M1) de la mâchoire inférieure de $M$. arvalis est caractérisée par la présence de cinq triangles d'émail bien individualisés alors que celle de $P$. subterraneus en compte trois bien individualisés et deux qui communiquent largement entre eux (Fig. 1) (Frechkop, 1958 ; Husson, 1962 ; Saint Girons, 1972, 1973a; Van Den Brink, 1967).

Par contre, si les mandibules font défaut - ce qui est habituel dans les débris de pelotes de réjection de rapaces - il conviendra d'utiliser un autre critère différenciant les deux espèces de crânes. Il n'est en effet pas possible de les distinguer au moyen des crêtes d'émail des molaires supérieures.

Actuellement, trois caractères sont essentiellement employés pour opérer cette distinction :

- Saint Girons (1972) se base sur la forme des sutures nasal-frontal et prémaxillaire-frontal (Fig. 2). Chez Microtus arvalis, la suture nasal-frontal est

Mammalia, t. 43, n०1, 1979. 
étroite et forme une pointe dirigée vers l'avant. Au contraire, chez Pitymys subterraneus, elle est large et ne forme aucune pointe dirigée vers l'avant.

- Husson (1962) mentionne la différence des rapports hauteur du crâne/ longueur du diastème. Ce rapport serait toujours supérieur à $93 \%$ chez Microtus arvalis et aurait une moyenne de $84,5 \%$ chez Pitymys subterraneus.

- Schmidt (1966) propose de considérer la largeur de la constriction interorbitaire, qui serait faible chez Microtus arvalis (de 3,1 à $3,8 \mathrm{~mm}$; moyenne : $3,4 \mathrm{~mm}$ ) et plus importante chez Pitymys subterraneus (de 3,3 à $4,2 \mathrm{~mm}$; moyenne : $3,8 \mathrm{~mm}$ ).

Dans cette note, nous nous proposons d'examiner successivement ces trois critères et de discuter de leur valeur respective.

\section{MÉthodes}

Le matériel qui a servi à cette étude provient de pelotes de réjection de rapaces nocturnes : chouette effraie, Tyto alba (Scopoli), et hibou moyen-duc, Asio otus (Linné). Ces pelotes ont été trouvées dans le sud de la Belgique en 1973 et 1974. La figure 3 situe les différents endroits de récolte.

2334 crânes de campagnols (Microtus arvalis $=1799$; Pitymys subterraneus $=535$ ) ont été identifiés sur base des crêtes d'émail de la $\mathbf{M}_{1}$ inférieure.

Pour vérifier l'hypothèse de Saint Girons (1972) ils ont été étudiés à la loupe et sériés quant à la forme des sutures.

Pour vérifier les hypothèses de Husson (1962) et de Schmidt (1966), ils ont été mesurés au $1 / 20^{\mathrm{e}}$ de $\mathrm{mm}$ au moyen d'un pied à coulisse.

\section{Résultats et conclusions}

\section{a) Examen des sutures nasal-frontal et prémaxillaire-frontal.}

Sur un total de 834 crânes de Microtus examinés, 22 ex. ne présentaient aucune échancrure importante dans le tracé de la suture nasal-frontal (Fig. 2) et pouvaient sur base de ce critère être confondus avec Pitymys subterraneus. Le $\chi^{2}$ calculé pour Microtus arvalis s'élève à 0,58 .

Chez Pitymys subterraneus, 5 ex. sur les 280 observés ont une suture nasalfrontal dont le tracé diffère assez nettement de celui mentionné par Saint Girons et possèdent d'autre part des tracés semblables entre eux. La fig. 4 montre le dessin de cette suture chez un des 5 ex. Le $\chi^{2}$ calculé pour Pitymys subterraneus égale 0,09 .

Ces valeurs du $\chi^{2}$ calculé sont nettement inférieures au $\chi^{2}$ théorique qui pour un risque d'erreur de $5 \%$ vaut 3,84 . Statistiquement parlant, l'utilisation de ce caractère convient donc comme critère de discrimination entre les deux espèces.

b) Analyse des rapports hauteur du crâne/longueur du diastème.

Ce critère doit être utilisé avec prudence quand on sait que la mesure de la hauteur du crâne est malaisée à prendre correctement. 


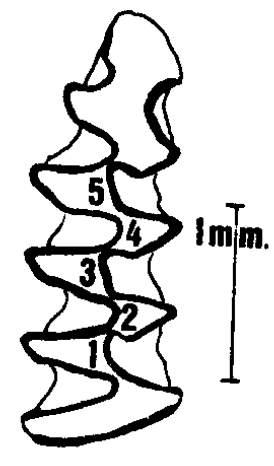

M.a.

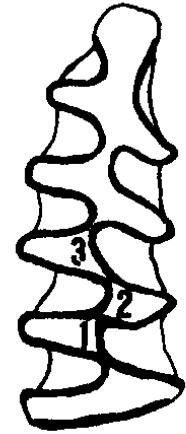

P.s.

Fig. 1. - Vue occlusale de la première molaire inférieure droite de Microtus arvalis (M.a.) et de Pitymys subterraneus (P.s.).

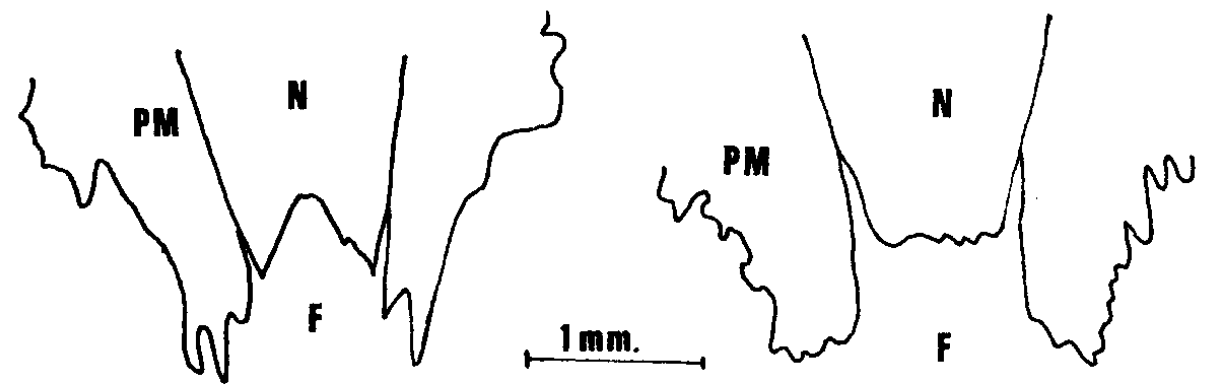

M.

P.s.

Fig. 2. - Suture nasal (N) - frontal (F) et frontal-prémaxillaire (PM) de M. arvalis (M.a.) et $P$. subterraneus (P.s.). (D'après Libois, 1975).

L'état des crânes de 920 Microtus arvalis et de 315 Pitymys subterraneus a permis l'analyse de ce rapport.

Les résultats en sont consignés dans la fig. 5. En abscisse, on trouvera les différentes classes exprimées en \% du rapport hauteur du crâne/longueur du diastème. En ordonnée figure le nombre d'individus par classe, exprimé en $\%$ du total des individus mesurés.

Les moyennes : 95,1\% pour Microtus arvalis et $85,7 \%$ pour Pitymys subterraneus sont significativement différentes. Par contre, l'étalement des mensurations est tel que pour des valeurs du rapport comprises entre 86 et $95 \%$, la discrimination n'est pas valable. Ce caractère ne devra donc être employé qu'en tenant compte de ces restrictions.

En guise de conclusion, nous dirons que le crâne de $M$. arvalis est moins comprimé dorso-ventralement que celui de $P$. subterraneus. La fig. 6 illustre ce dernier fait. Toutefois seules les valeurs extrêmes du rapport envisagé sont tout-à-fait discriminatoires. 


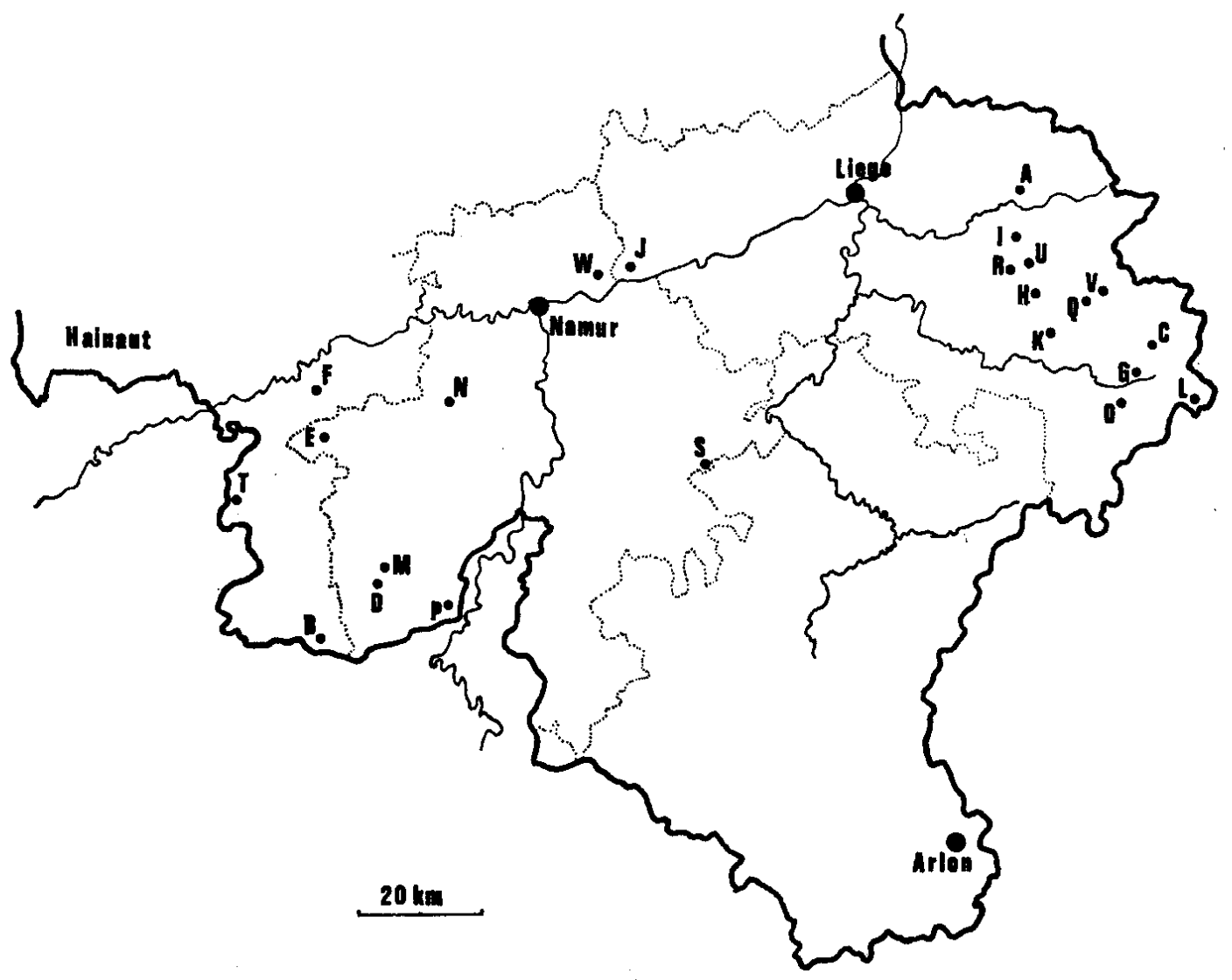

Fig. 3. - Carte de répartition des points de récolte des échantillons. Les lettres renvoient au tableau 1.

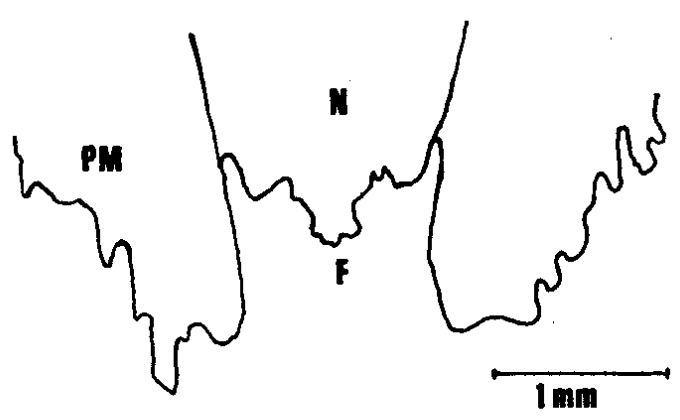

Fig. 4. - Suture nasal (N)-frontal (F) et frontal-prémaxillaire (PM) d'un P. subterraneus aberrant ".

c) Etude de la largeur de la constriction interorbitaire.

Nous avons mesuré la largeur de la constriction interorbitaire de 1799 Microtus arvalis et de 535 Pitymys subterraneus (voir tableau 1). Les résultats se trouvent dans la figure 7. La largeur de la constriction interorbitaire de $M$. arvalis est comprise entre 2,60 et $3,50 \mathrm{~mm}$ et a une valeur moyenne de $3,09 \mathrm{~mm}$, tandị qu'elle varie de 3,30 à $4,00 \mathrm{~mm}$ chez $P$. subterraneus avec une 


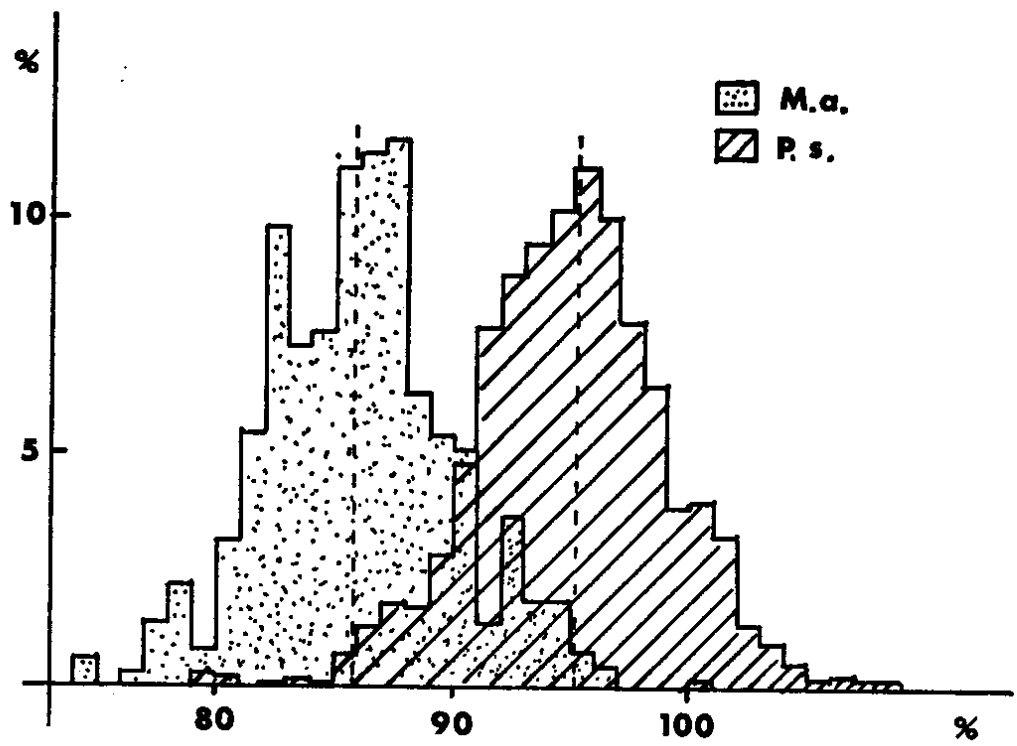

Fig. 5. - Rapports hauteur du crâne/longueur du diastème : - de $M$. arvalis (M.a.) : $\mathrm{n}=920 ; \mathrm{m}=95,1 \% ; \mathrm{s}=1,270 ;-$ de $P$. subterraneus $($ P.s. $): \mathrm{n}=315 ; \mathrm{m}=85,7 \%$; $\mathrm{s}=0,687$.

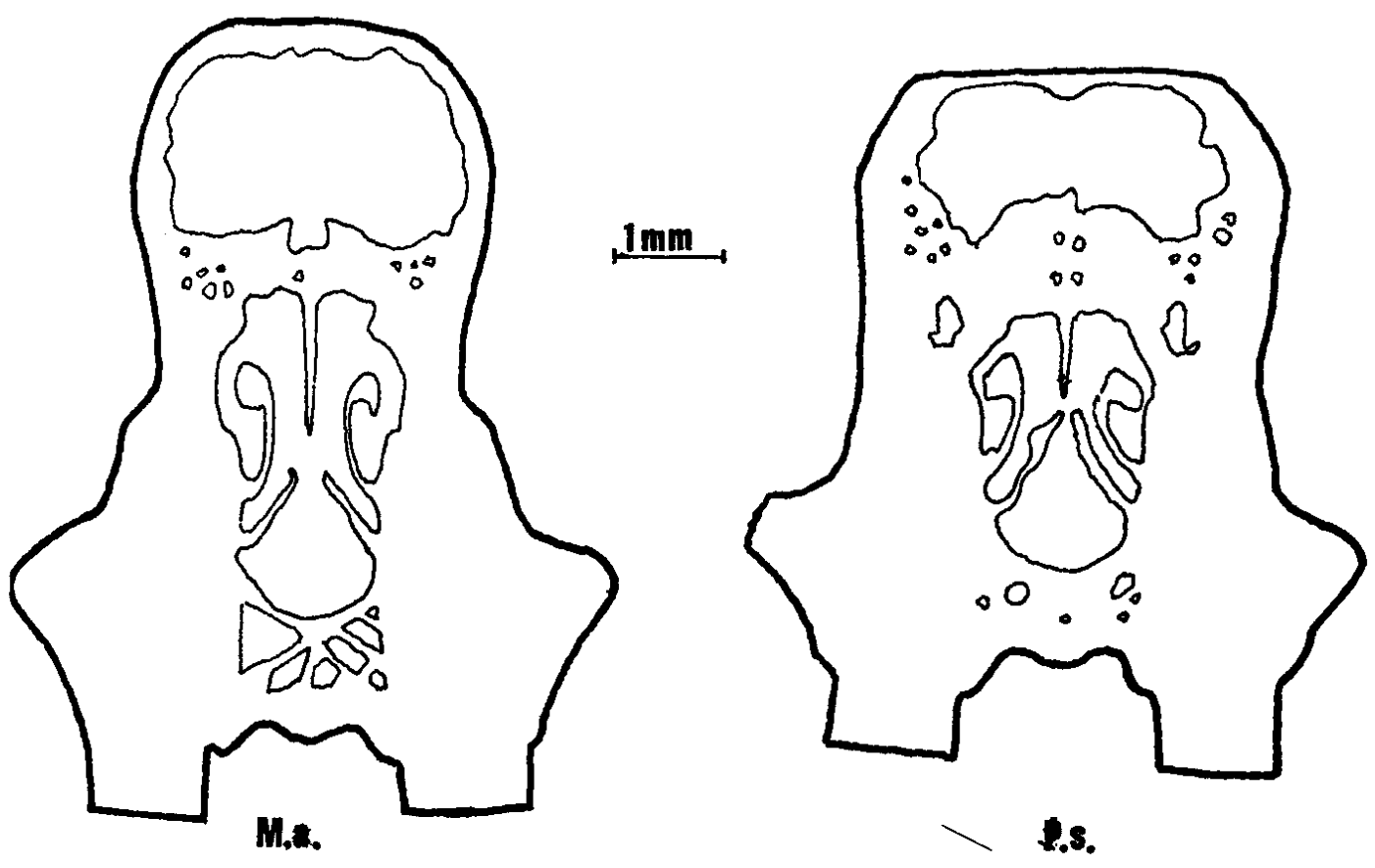

Fig. 6. - Schémas (d'après cliché J. L. de Sloover) de coupes transversales du crâne de $M$. arvalis et de $P$. subterraneus au niveau de la $M 1$ sup. Ces coupes ont été obtenues après inclusion des crânes dans du polystyrène et polissage jusqu'à l'épaisseur désirée (préparation : P. Overlau et R. Libois).

valeur moyenne de $3,65 \mathrm{~mm}$. La différence entre ces moyennes est hautement significative. Toutefois, il existe une faible zone de recouvrement des données entre les mesures 3,30 et $3,50 \mathrm{~mm}$. 
TABLEAU 1. - Origine et caractéristiques des échantillons de crânes. $\mathbf{n}:$ nombre de crânes mesurés ; $m$ : largeur moyenne de la constriction interorbitaire ; s : écart-type.

\begin{tabular}{|c|c|c|c|c|c|c|c|}
\hline \multirow[t]{2}{*}{ Localités } & \multirow[t]{2}{*}{ code } & \multicolumn{3}{|c|}{ Microtus arvalis } & \multicolumn{3}{|c|}{ Pitymys subterraneus } \\
\hline & & $\mathrm{n}$ & $\mathrm{m}$ & $\mathbf{s}$ & n & $\mathrm{m}$ & $\mathbf{s}$ \\
\hline Baelen & $\mathrm{A}$ & 7 & - & - & 1 & - & - \\
\hline Baileux & B & 2 & - & - & 12 & - & - \\
\hline Bullingen & C & 168 & 3,15 & 0,139 & 9 & - & - \\
\hline Couvin & $\mathbf{D}$ & 374 & 3,02 & 0,132 & 116 & 3,62 & 0,126 \\
\hline Fontenelle & $\mathbf{E}$ & 96 & 3,05 & 0,106 & 35 & 3,69 & 0,132 \\
\hline Gozée & $\mathbf{F}$ & 1 & - & - & 3 & - & - \\
\hline Heppenbach & G & 10 & - & - & 1 & - & - \\
\hline Hockai & $\mathrm{H}$ & 25 & - & - & 9 & - & - \\
\hline Jalhay & 1 & 238 & 3,17 & 0,106 & 6 & - & - \\
\hline Landenne & $J$ & 396 & 3,09 & 0,138 & 239 & 3,67 & 0,110 \\
\hline Malmedy & $\mathrm{K}$ & 31 & - & - & 8 & - & - \\
\hline Manderfeld & $\mathrm{L}$ & 33 & - & - & 5 & - & - \\
\hline Mariembourg & $\mathbf{M}$ & 19 & - & - & 7 & - & - \\
\hline Mettet & $\mathbf{N}$ & 84 & 3,05 & 0.121 & 2 & - & - \\
\hline Meye rode & 0 & 2 & - & - & - & - & - \\
\hline Oignies & $\mathbf{P}$ & 9 & - & - & 5 & - & - \\
\hline Ovifat & $Q$ & 1 & - & - & 1 & - & - \\
\hline Sart-1ez-Spa & $\mathbf{R}$ & - & - & - & 20 & - & - \\
\hline Serinchamps & $\mathrm{s}$ & 218 & 3,06 & 0,133 & 35 & $\cdot 3,62$ & 0,105 \\
\hline Sivry & $T$ & 23 & - & - & 8 & - & - \\
\hline Solwaster & U & 37 & - & - & 8 & - & - \\
\hline Sourbrodt & $\mathrm{V}$ & 24 & - & - & 2 & - & - \\
\hline Vezin & W & 1 & - & - & 3 & - & - \\
\hline TOTAL & - & 1799 & 3.09 & 0,255 & 535 & 3,65 & 0,118 \\
\hline
\end{tabular}

Nous avons poussé plus loin l'analyse des échantillons des populations dont nous possédions un certain nombre de crânes, à savoir celles des points $\mathrm{C}, \mathrm{D}$, $\mathrm{E}, \mathrm{I}, \mathrm{J}, \mathrm{N}$ et $\mathrm{S}$ (fig. 3 ).

Les mesures se rapportant à $M$. arvalis sont consignées dans la fig. 8 , tandis que celles relatives à $P$. subterraneus se trouvent dans la fig. 9 .

Pour le caractère envisagé, deux faits se dégagent de l'examen des figures 8 et 9 :

- D'une part, au niveau de chaque endroit de récolte, il existe une différence très nette entre les deux espèces comme pour la figure récapitulative 7 .

- D'autre part, il y a une ressemblance entre les différentes populations de chaque espèce (voir fig. 10). Cependant, les échantillons de l'est de la 


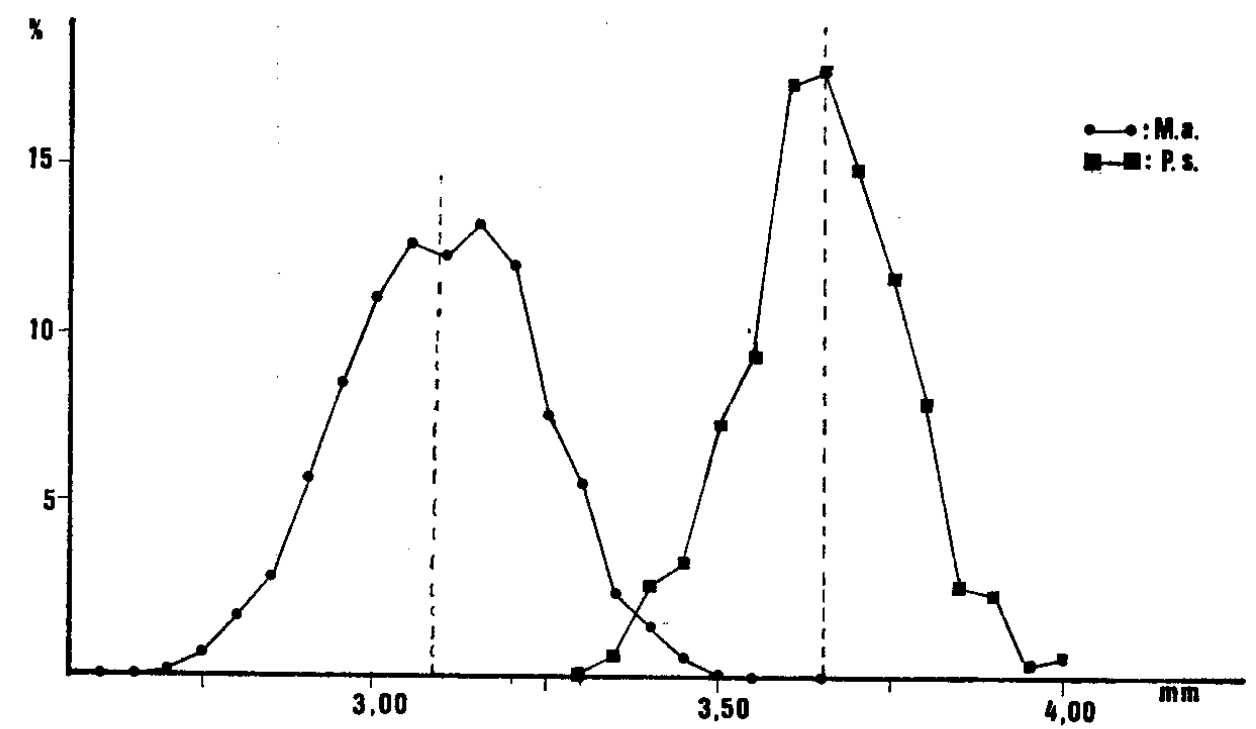

Fig. 7. - Largeur de la constriction interorbitaire de $M$. arvalis (M.a.) et $P$. subterraneus (P.s.).

Belgique (soit $\mathrm{C}$ et $\mathrm{I}$ ), dont les moyennes sont semblables entre elles, ont une moyenne significativement supérieure à celle de tous les autres.

En outre, les échantillons tant de $M$. arvalis que de $P$. subterraneus récoltés au point D (sud de l'Entre-Sambre et Meuse) montrent une valeur moyenne inférieure à celle des autres échantillons.

Ce caractère a donc une bonne valeur discriminatoire.

Schmidt (1966), dans une étude portant sur des individus provenant des Karpates et du plateau de Transylvanie, trouve pour ce même caractère des valeurs s'échelonnant de 3,1 à $3,7 \mathrm{~mm}$ chez $M$. arvalis (moyenne : $3,4 \mathrm{~mm}$ ) et de 3,3 à $4,2 \mathrm{~mm}$ chez $P$. subterraneus (moyenne : $3,8 \mathrm{~mm}$ ). La différence entre ces moyennes est hautement significative.

Cependant, les valeurs obtenues par Schmidt sont aussi hautement significativement différentes de celles enregistrées par nous en Belgique, et cela aussi bien en ce qui concerne $M$. arvalis que $P$. subterraneus. Au vu de la fig. 11 , il apparait même que ces mesures se superposent, ce qui au premier abord est assez déroutant. Force est donc de conclure que les populations karpatiques de ces deux espèces de campagnols diffèrent des belges par la largeur de la constriction interorbitaire.

L'explication de ce phénomène réside peut-être dans l'hypothèse de Mayr : "Pratiquement toutes les espèces continentales ayant une répartition vaste et continue présentent sur un ou plusieurs caractères morphologiques une variation d'ordre clinal " (Mayr in Blondel, 1967).

$M$. arvalis et $P$. subterraneus étant deux espèces continentales à répartition vaste et continue, il est probable que certains de leurs caractères présentent une variation clinale.

Il en résulte qu'il serait absurde de vouloir distinguer $M$. arvalis de $P$. subterraneus par ce caractère sans tenir compte de la provenance des échantillons à identifier ni sans disposer antérieurement de données valables relatives à ce caractère pour les populations d'où sont issus les échantillons. 

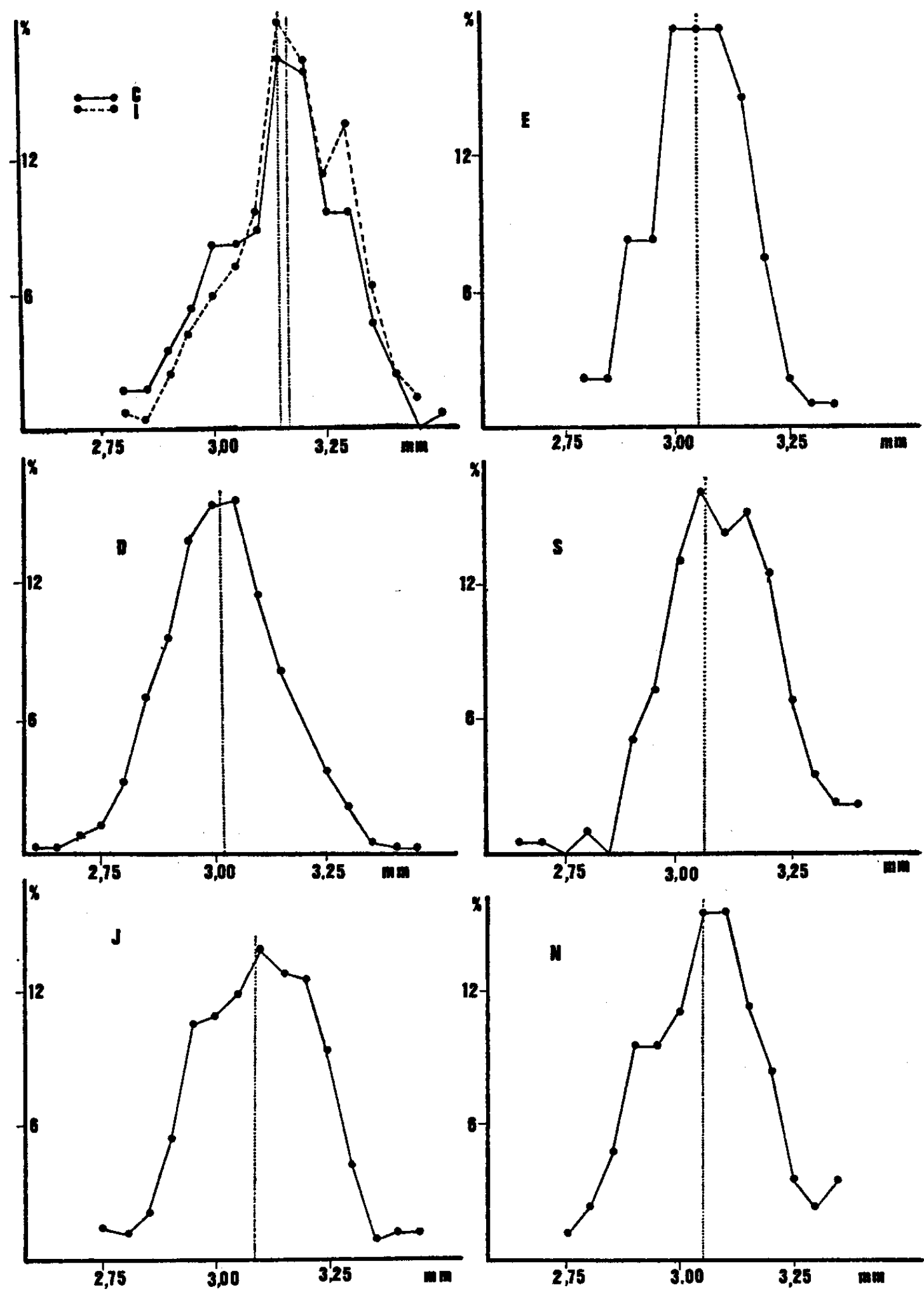

Fig. 8. - Largeur de la constriction interorbitaire de 7 échantillons de Microtus arvalis. Les lettres renvoient au tableau 1. 

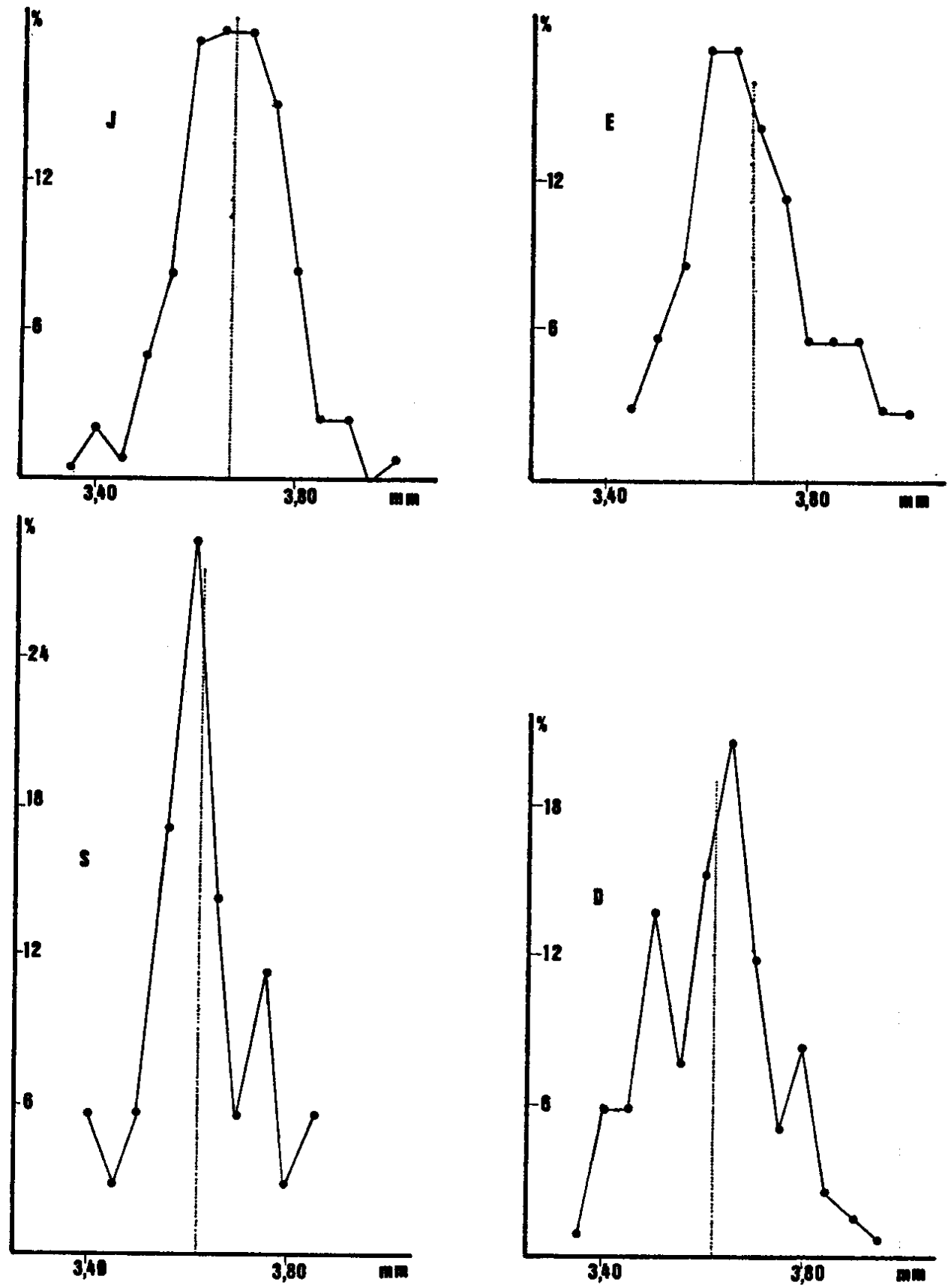

Fig. 9. - Largeur de la constriction interorbitaire de 4 échantillons de Pitymys subterraneus. Les lettres renvoient au tableau 1.

Nous aurions enfin désiré savoir si la largeur de la constriction interorbitaire variait en fonction de l'âge chez ces deux campagnols. Pour tenter de résoudre le problème, nous avons appliqué deux méthodes. D'une part, nous avons piégé un certain nombre d'animaux, déterminé leur âge par la méthode de la pesée du cristallin : méthode de Lord (Martinet, 1966 ; Le Louarn, 1971) et tenté d'établir une corrélation avec la largeur interorbitaire. Pour $11 M$. 
arvalis, nous avons calculé un coefficient de corrélation de 0,32 , tandis que pour $37 P$. subterraneus, il s'est avéré égal à 0,24 .

D'autre part, nous basant sur la méthode employée par Saint Girons $(1965$ et $1973 b)$ qui permet de distinguer les classes d'âge par la mesure de la longueur du diastème, nous avons étudié la largeur de la constriction interorbitaire de chaque espèce pour chaque classe d'âge (fig. 12 et 13).

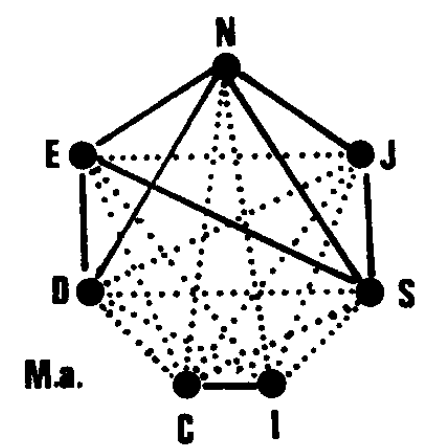

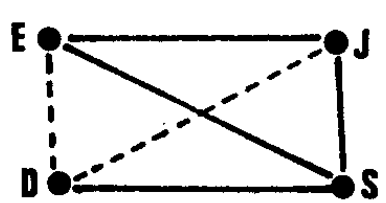

P.s.

Fig. 10. - Comparaison statistique des largeurs moyennes de la constriction interorbitaire des différents échantillons belges de $M$. arvalis (M.a.) et $P$. subterraneus (P.s.) Traits continus : moyennes identiques; traits interrompus : moyennes significativement différentes $(p=0,01)$ (test de Scheffé). Les lettres renvoient au tableau 1.

(N.B. : Dans la figure de gauche, les points $\mathrm{E}$ et $\mathrm{J}, \mathrm{D}$ et $\mathrm{S}$ devraient être reliés par un trait continu.)

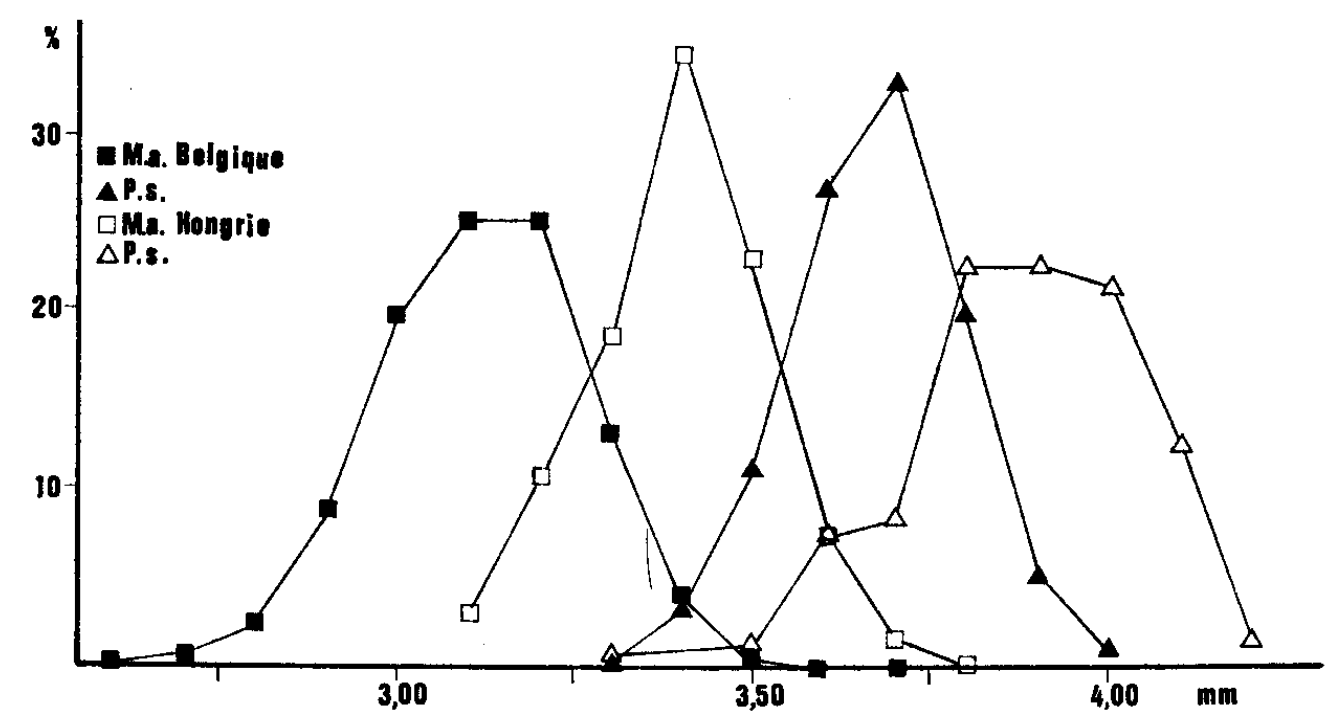

Fig. 11. - Largeur de la constriction interorbitaire de $M$. arvalis et $P$. subterraneus en Hongrie (d'après Schmidt, 1966) et en Belgique.

Chez $M$. arvalis, 5 classes sont définies : jeunes non sevrés (I), juvéniles (II), subadultes (III), adultes (IV) et vieux adultes (V). Seule la moyenne de la classe II est significativement différente de celle de la classe V (test de Scheffé).

En ce qui concerne $\boldsymbol{P}$. subterraneus, Saint Girons distingue 3 classes : jeunes (I), adultes (II) et vieux adultes (III). Les moyennes calculées pour ces différentes classes ne sont pas significativement différentes. 
TABLEAU 2. - Largeur de la constriction interorbitaire en fonction de l'âge.

Classe d'âge

$\begin{array}{crrc} & \text { n } & \text { m } & \text { s } \\ \text { I } & 29 & 3,10 & 0,125 \\ \text { II } & 523 & 3,09 & 0,143 \\ \text { III } & 322 & 3,11 & 0,141 \\ \text { IV } & 560 & 3,12 & 0,136 \\ \text { V } & 109 & 3,14 & 0,147\end{array}$

P. subterraneus

$\begin{array}{ccc}\text { n } & \text { m } & \text { s } \\ 200 & 3,66 & 0,114 \\ 173 & 3,64 & 0,117 \\ 53 & 3,65 & 0,125 \\ - & - & -\end{array}$
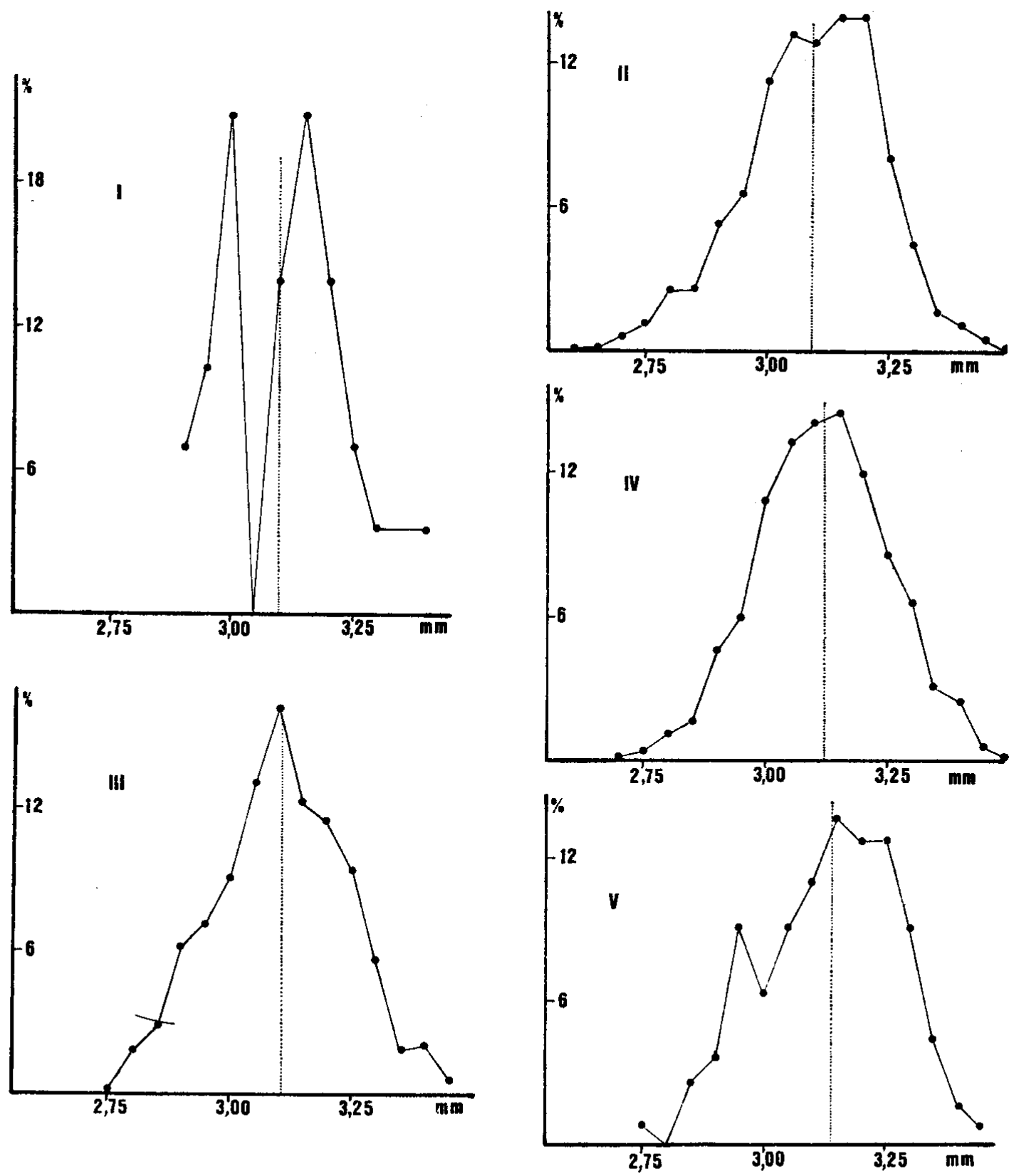

Fig. 12. - Largeur de la constriction interorbitaire des 5 classes d'âge définies chez $M$ arvalis. 

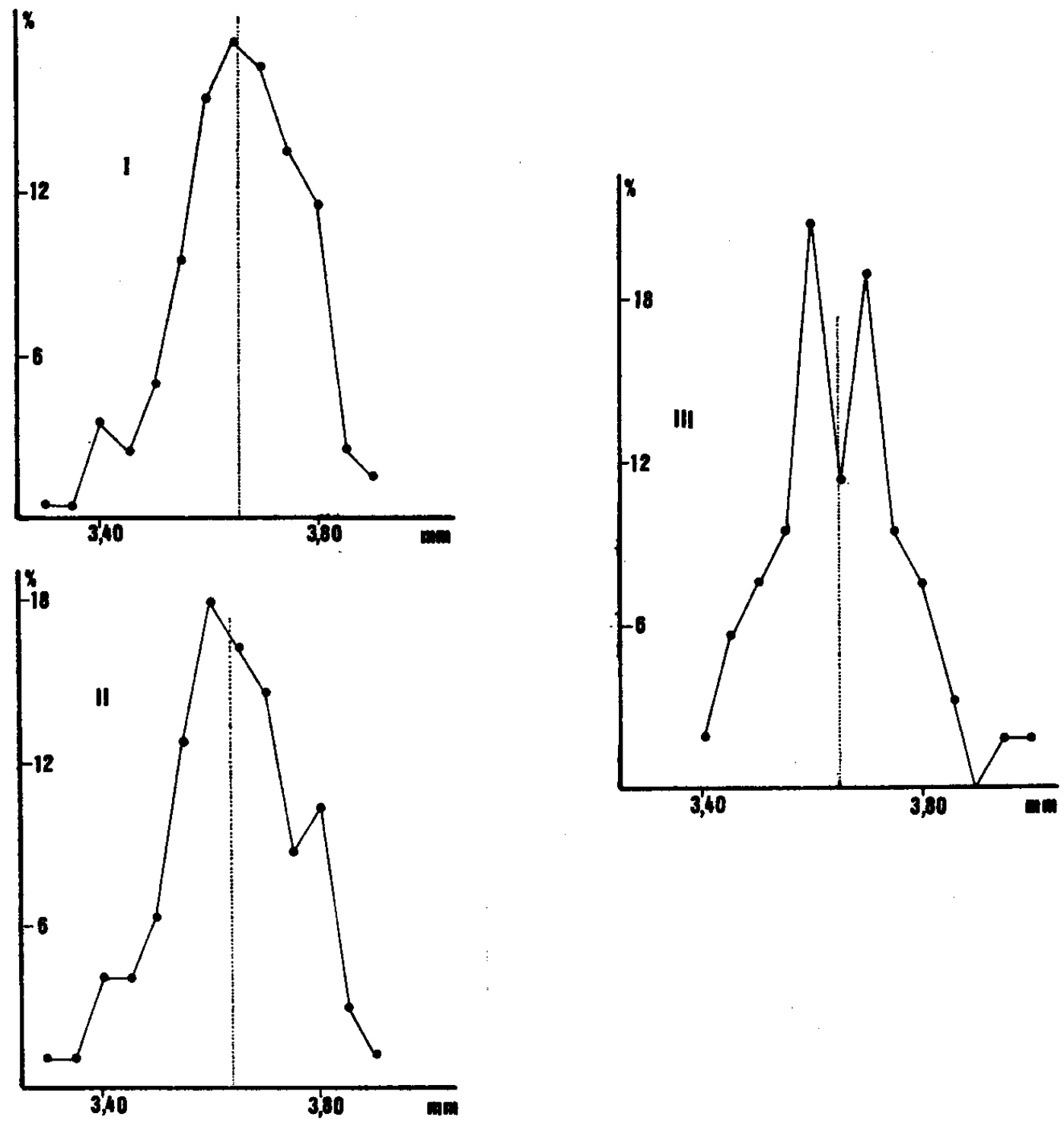

Fig. 13. - Largeur de la constriction interorbitaire des 3 classes d'âge définies chez $P$. subterraneus.

Il n'y a donc pas de corrélation étroite entre l'âge des animaux et la largeur de la constriction interorbitaire, bien que chez les campagnols des champs âgés une crête apparaisse au niveau du rétrécissement interorbitaire. Ce critère pourra en conséquence être utilisé dans la discrimination des deux espèces étudiées quel que soit l'âge des animaux à identifier.

Ces conclusions rejoignent celles de Schmidt : il avait pu établir que la largeur de la constriction interorbitaire varie probablement peu avec l'âge des animaux, du moins à partir du moment où les jeunes campagnols se promènent en surface et sont susceptibles d'être capturés par les rapaces.

\section{REMERCIEMENTS}

Au terme de ce travail, il m'est particulièrement agréable de remercier MM. J. Bernard et J. Tahon, respectivement Directeur et Chef de travaux à la Station de zoologie appliquée de Gembloux, qui m’ont précieusement aidé et conseillé et qui ont accepté de relire mon manuscrit. 
Je suis également redevable à MM. P. Overlau et J.-L. de Sloover, Professeurs aux Facultés universitaires de Namur, pour leur collaboration dans la réalisation et la photographie des lames minces.

Ma reconnaissance s'adresse aussi à mon oncle, M. J. Libois, qui a supporté mes nombreuses allées et venues dans ses campagnes lors des piégeages, ainsi qu'aux personnes qui m'ont aimablement fait parvenir des pelotes de réjection : MM. J. Doucet, A. Jacquet et A. Magerat, Mlle D. Plouvier et MM. P. Plouvier, J. L. Rousseau, F. Scheuren et E. Sérusiaux.

\section{SUMMARY}

In order to identify skulls of field voles, Microtus arvalis (Pallas), and of pine voles Pitymys subterraneus (de Sélys Longchamps), when the mandibles are absent, 1799 skulls of field voles and 535 skulls of pine voles from different Belgian populations have been studied in three aspects :

- The pattern of the nasal-frontal and premaxillary-frontal sutures. This pattern is an excellent criterion by which identify the two species, and it is almost always accurate.

- The value of ratios between a skull's height and the length of its diastema, This technique enables one to discriminate between field vole specimens with ratios above $95 \%$ and pine vole specimens below $86 \%$.

- The width of the interorbital constriction. This seems to be an equally good criterion of identification, even for young animals. However in Europe it seems to have a clinal variation, a finding which should be verifled. For the Belgian populations studied, the values for the field vole range from 2.60 to $3.50 \mathrm{~mm}$ (mean $3.09 \mathrm{~mm}$ ). In the pine vole they range from 3.30 to $4.00 \mathrm{~mm}$ (mean $3.65 \mathrm{~mm}$ ).

Laboratoire d'Ethologie et Psychologie animales, Institut de Zoologie de l'Université, 22, quai Van Beneden, B-4020 Liege. Station Scientifique des Hautes-Fagnes, Mont-Rigi, B-4898 Robertville.

\section{BIBLIOGRAPHIE}

Blondel, J., 1967. - Synécologie des passereaux et migrateurs dans le midi méditerranéen français. Centre régional de documentation pédagogique, Marseille, $239 \mathrm{pp}$.

Frechкop, S., 1958. - Faune de Belgique : Mammiferes. I.R.Sc.N.B. Bruxelles, 545 pp.

Husson, A. M., 1962. - Het determineren van schedelresten van zoogdieren in braakballen van uilen. Zool. bijdr. $n r .5$, Rijksmuseum van natuurlijke Historie, Leiden, $63 \mathrm{pp}$.

Le Louarn, H., 1971. - Détermination de l'âge par la pesée du cristallin chez quelques espèces de rongeurs. Mammalia, 35 : 636-643.

Libors, R. M., 1975. — La détermination des micromammifères belges (Chiroptères exceptés) en main et d'après les restes crâniens présents dans les pelotes de réjection de rapaces. Natural. belges, $56: 165-188$.

Martinet, L., 1965. - Détermination de l'âge chez le campagnol des champs (Microtus arvalis) par la pesée du cristallin. Mammalia, $30: 425-430$.

Sarnt Grrons, M.-C., 1965. - Notes sur les Mammifères de France. IV : Prélèvements exercés sur des populations de petits mammifères par la chouette effraie (Tyto alba) (région de Lyon). Mammalia, 29 : 42-53.

- 1972. - La reconnaissance des proies contenues dans les pelotes de rapaces en France, Belgique, Pays-Bas et Luxembourg. Overdruk publicaties van het natuurhistorisch Genootschap in Limburg, Reeks XXII, affievering, 1, 2, 3 en 4.

- 1973a. - Les mammiferes de France et du Benelux. Doin, Paris, 481 pp.

- $\quad 1973 b$. - L'âge des mammifères dans le régime de deux rapaces nocturnes : Tyto alba et Asio otus. Mammalia, 37 : 439-456. 
Schmidt, E., 1966. - Die Rolle der interorbitalen Breite bei der Unterscheidung aus Eulengewöllen stammender Schädel von Microtus arvalis und Pitymys subterraneus. Zeits. Säugetierkunde, 31 : 324-327.

Van Den Brink, F. H., 1967. - Guide des mammifères sauvages d'Europe occidentale. Delachaux et Niestlé, Neuchâtel, 263 pp. 